\title{
BMJ Open Recommendations for integrating physiotherapy into an interprofessional outpatient care setting for people living with HIV: a qualitative study
}

Heather deBoer, ${ }^{1}$ Stephanie Cudd, ${ }^{1}$ Matthew Andrews, ${ }^{1}$ Ellie Leung, ${ }^{1}$ Alana Petrie, ${ }^{1}$ Soo Chan Carusone, ${ }^{2,3}$ Kelly K O’Brien ${ }^{1,4}$

To cite: deBoer $\mathrm{H}$,

Cudd S, Andrews M, et al. Recommendations for integrating physiotherapy into an interprofessional outpatient care setting for people living with HIV a qualitative study. BMJ Open 2019;9:e026827. doi:10.1136/ bmjopen-2018-026827

- Prepublication history for this paper is available online. To view these files, please visit the journal online (http://dx.doi org/10.1136/bmjopen-2018026827).

Received 21 September 2018 Revised 8 March 2019 Accepted 12 April 2019

Check for updates

(C) Author(s) (or their employer(s)) 2019. Re-use permitted under CC BY-NC. No commercial re-use. See rights and permissions. Published by BMJ.

${ }^{1}$ Department of Physical Therapy, University of Toronto, Toronto, Ontario, Canada

${ }^{2}$ Casey House, Toronto, Ontario, Canada

${ }^{3}$ Department of Research Methods, Evidence, and Impact, McMaster University, Hamilton, Ontario, Canada

${ }^{4}$ Institute of Health Policy, Management and Evaluation (IHPME); Rehabilitation Sciences Institute (RSI), University of

Toronto, Toronto, Ontario, Canada

Correspondence to

Dr Kelly K 0'Brien;

kelly.obrien@utoronto.ca

\section{ABSTRACT}

Objectives To identify factors to consider when integrating physiotherapy (PT) into an interprofessional outpatient HIV care setting from the perspective of healthcare professionals and adults living with HIV. Design We conducted a qualitative descriptive study using semi-structured interviews (healthcare professionals) and focus groups (adults living with HIV). We asked participants their perspectives on barriers, facilitators and strategies to accessing and participating in outpatient PT, important characteristics physiotherapists should possess working in outpatient HIV care, content and structure of PT delivery, and programme evaluation. Recruitment and setting We purposively sampled healthcare professionals based on their experiences working in interprofessional HIV care and recruited adults with HIV via word of mouth and in collaboration with an HIV-specialty hospital in Toronto, Canada. Interviews were conducted via Skype or in-person and focus groups were conducted in-person at the HIV-specialty hospital.

Participants 12 healthcare professionals with a median of 12 years experience in HIV care, and 13 adults living with HIV (11 men and 2 women) with a median age of 50 years and living with a median of 6 concurrent health conditions in addition to HIV.

Results Overall impressions of PT in outpatient HIV care and factors to consider when implementing PT into an interprofessional care setting include: promoting the role of, and evidence for, PT in outpatient HIV care, structuring PT delivery to accommodate the unique needs and priorities of adults living with HIV, working collaboratively with a physiotherapist on the healthcare team and evaluating rehabilitation as a component of interprofessional care.

Conclusions Multiple factors exist for consideration when implementing PT into an interprofessional outpatient HIV care setting. Results provide insight for integrating timely and appropriate access to evidence-informed rehabilitation for people living with chronic and episodic illness, such as HIV.

\section{INTRODUCTION}

Due to healthcare advances and improvements in combination antiretroviral therapy, people living with HIV are experiencing
Strengths and limitations of this study

- To our knowledge, this is the first study to explore the role of and factors to consider when implementing physiotherapy into an interprofessional outpatient HIV care setting.

- Exploring perspectives from adults living with HIV and healthcare professionals using multiple methods of data collection (focus groups and interviews) enabled us to gather perspectives and recommendations from a diverse stakeholder group involved in accessing and delivering HIV care to develop recommendations for integrating physiotherapy into an interprofessional outpatient HIV care setting.

- Healthcare providers and those involved in programme development can use results from this study when developing or adapting interprofessional outpatient programmes for adults living with HIV and multimorbidity.

- This study was conducted in collaboration with a HIV-specialty hospital in an urban Canadian city and therefore, results may not be transferable to low-income to middle-income countries or rural or remote areas.

- This study specifically focuses on an interprofessional outpatient programme for adults living with HIV; further study is necessary to determine the relevance of results to similar populations, such as those living with other chronic conditions and multimorbidity.

increased life expectancy and chronicity of ageing and multimorbidity. ${ }^{1-3}$ Authors of a cross-sectional population-based study in Ontario reported that adults living with HIV experienced increased prevalence of mental and physical medical conditions, and multimorbidity, defined as the presence of several chronic conditions compared with the general population. ${ }^{4}$ Many individuals experience disability associated with HIV and multimorbidity, defined as fluctuations in health, including physical, cognitive, mental or emotional symptoms and impairments, 
difficulties carrying out day-to-day activities, challenges related to social inclusion and uncertainty about future health. ${ }^{5}$ Rehabilitation, including physiotherapy (PT), has a role in managing and minimising the spectrum of disability experienced by people living with HIV. ${ }^{6}$ Evidence suggests that PT can help to improve functional mobility, ${ }^{7}$ pain management, ${ }^{8}$ peripheral neuropathy ${ }^{9}$ and address impairments associated with ageing among older adults living with HIV and complex comorbidities. ${ }^{10}{ }^{11}$ Further high-level evidence specifically demonstrated the benefits of exercise interventions among adults living with HIV. ${ }^{12} 13$

Despite evidence supporting the role and benefits, few people with HIV access PT services. ${ }^{14}$ Barriers to accessing PT among adults living with HIV include lack of available services, stigma, financial barriers and lack of knowledge among healthcare professionals about the role of rehabilitation in HIV care. ${ }^{15}$ As HIV transitions from a palliative to chronic illness, novel approaches to PT care delivery may help to overcome barriers accessing PT for people living with complex chronic illness. Authors of a South African study advocated for home-based and community-based PT in order to address financial barriers and mobility limitations. ${ }^{16}$ Casey House, an HIV-specialty hospital in Toronto, opened a publicly funded day health programme in 2017 with the goal of improving access and coordination of interprofessional health services for people living with HIV. ${ }^{17}$ To our knowledge, this is the first to include PT services and offers a foundation for considering rehabilitation as part of an interprofessional team approach in an outpatient HIV care setting. Despite emerging outpatient PT-focused programming and services for people living with HIV, to our knowledge no criterion or recommendations exist for implementing PT as an interprofessional element of outpatient HIV care.

Authors of a qualitative study described the role of PT in addressing physical, psychological and social aspects of health from the perspective of people living with HIV and health professionals with experience in HIV care. Results highlighted the role of PT as multidimensional and client-centred and identified eight contextual factors important to consider in interprofessional HIV care that included: ageing, episodic nature of HIV, multimorbidity, competing priorities, continuity of care, stigma, resource security and social isolation. ${ }^{18}$ These factors, while complex are important to consider as evidence to inform how to best integrate PT within an interprofessional outpatient HIV care setting. Interprofessional care is well established as a valuable component of coordinated, comprehensive HIV care. ${ }^{19-21}$ However, specific recommendations for how to integrate PT into an interprofessional outpatient HIV programme are currently lacking. Hence, the purpose of this study was to identify factors to consider when integrating PT into an interprofessional outpatient HIV care setting from the perspective of healthcare professionals and people living with HIV.

\section{METHODS}

\section{Study design}

We conducted a qualitative descriptive study comprised of interviews with healthcare professionals, and focus groups with adults living with HIV. ${ }^{22}$ In this study, we used the day health programme at Casey House, a community-based HIV-specialty hospital in Toronto, Ontario, as an exemplar to focus on factors to consider when integrating PT into an interprofessional outpatient setting for adults living with HIV. ${ }^{17}{ }^{18}$ Casey House provides a continuum of interdisciplinary healthcare services including inpatient and outpatient (day health programme) care and community outreach services for people living with HIV and complex multimorbidity. Services may include but are not limited to, medicine, nursing, social work, mental health and substance use services, recreation therapy, massage therapy and most recently, PT making this an ideal setting in which to examine the integration of PT into an interprofessional outpatient care setting. ${ }^{23}$

\section{Patient and public involvement}

This research evolved from a longstanding community-academic-clinical partnership involving people living with HIV, clinicians and researchers who identified key research priorities in HIV and rehabilitation. This study addresses research priorities established by the Canada-International $H I V$ and Rehabilitation Research Collaborative (CIHRRC), a network of over 90 people living with HIV, researchers, clinicians, representatives from community organisations and policy stakeholders who collectively work to advance and translate HIV and rehabilitation research. ${ }^{24}$ CIHRRC conducted a multistakeholder consultation with researchers, people living with HIV, clinicians and community partners to establish a Framework of Research Priorities in HIV, Disability and Rehabilitation. ${ }^{25}$ This framework describes six priorities across three content areas: (1) exploring episodic health and disability; (2) effectiveness of rehabilitation interventions and models of service delivery and (3) advancing patient-reported outcome measures in HIV rehabilitation. ${ }^{25}$ This research specifically addresses priority \#2 examining models of rehabilitation service delivery in the context of HIV.

We consulted with a community member living with HIV who advised on the development of the data collection tools. Results from this study were translated in the form of a presentation with Casey House staff, and a fact sheet summary summarising the role of PT in HIV care and providing practical information of how to access PT. The fact sheet was emailed to study participants and more broadly disseminated via an openly accessible link on the CIHRRC website (http://cihrrc.hivandrehab.ca/docs/ Fact-Sheet-Where-How-PT-Fits-DHP-FINAL--Nov-15-17. pdf). ${ }^{18}$ Results from this study informed the integration of PT into the interprofessional outpatient care setting (Casey House day health programme), which serves as a foundation for a community-engaged evaluation of the process and outcomes of rehabilitation for people living with HIV and complex multimorbidity. ${ }^{23}$ 


\section{Recruitment}

Healthcare professionals

We recruited healthcare professionals who self-identified as experts in HIV care using purposive sampling, whereby authors (KKOB, SCC) identified known professionals working in the field. Healthcare professionals were defined as health providers who are registered or voluntarily designated by a governing body. To ensure we obtained perspectives from a variety of rehabilitation professionals with expertise in interprofessional HIV care across hospital and community settings, we purposively sampled and recruited rehabilitation professionals from Canada and the UK via the CIHRRC. ${ }^{24}$

\section{People living with HIV}

We recruited adults 18 years or older who self-identified as living with HIV via posters and word of mouth by Casey House clients and staff. Members of the research team identified themselves to potential participants as students in the Department of Physical Therapy at the University of Toronto who were advised by advisors throughout the research (KKOB and SCC). A member of the research team obtained written or verbal informed consent from each participant immediately prior to each interview or focus group.

\section{Data collection}

We developed semi-structured interview (healthcare professionals) and focus group (adults living with HIV) guides to explore considerations when integrating PT into interprofessional outpatient HIV care for adults, using the Casey House day health programme as an exemplar. A community member living with HIV with research expertise provided feedback on drafts of the interview and focus group discussion guides. Guiding questions were devised to explore perspectives in the following areas: strategies of how to enable access to an outpatient PT programme for people living with HIV, barriers and facilitators to adults living with HIV participating in an outpatient PT programme, characteristics of physiotherapists that are important for working in outpatient HIV care, recommendations for content and structure of PT sessions in order to accommodate the unique needs and priorities of people living with HIV and how to evaluate the PT programme in the context of an outpatient, interprofessional HIV care setting. We met as a research team throughout data collection to discuss overall impressions of the interviews and focus groups. We revised the interview guide five times and the focus group guide once during the course of data collection. We adapted the guides to improve clarity of the questions and expand on specifics related to evolving codes. This ongoing refinement helped to maximise our ability to elicit participant responses in subsequent interviews and focus groups in order to comprehensively describe factors for consideration when integrating PT into HIV care. ${ }^{18}$

We conducted and audio-recorded 12 face-to-face or Skype interviews with healthcare professionals and two focus groups at Casey House with adults living with HIV. Two research team members were present for each interview, and three were present for each focus group. One team member facilitated the interview (MA, SC, HD, AP) or focus group (MA) and others assisted with obtaining consent and documenting field notes (MA, SC, HD, EL, AP). We collected data either verbally postinterview (healthcare professionals) or via a self-administered questionnaire (adults living with HIV) to understand participant demographics, disease characteristics (adults living with HIV), experiences working in HIV care (healthcare professionals) and experiences with PT (adults living with HIV). Interview and focus group audio-recordings were transcribed verbatim and reviewed for accuracy. Further details on our methodology are published in a manuscript that describes the role of PT from the perspectives of adults living with HIV and healthcare professionals working in HIV care. ${ }^{18}$

\section{Data analysis}

We initially read the transcripts independently and noted context and first impressions. ${ }^{26}$ We then used a conventional content analytical approach to code the transcripts. ${ }^{27}$ Data were organised using NVivo V.10 software. ${ }^{28}$ All members of the team independently read and coded five of the same transcripts (three interviews and two focus groups), and met seven times to discuss overall impressions, coding and adaptations to guides for subsequent data collection. We developed a draft coding scheme based on the first four team-coded transcripts (two interviews and two focus groups) and ensured coding consistency with an additional team-coded interview. Pairs of two team members (HDB, MA, SC, EL, AP) independently coded the remaining transcripts and met to discuss coding and resolve discrepancies. ${ }^{18}$ We identified common responses and terms in transcripts, and then grouped related codes into themes to highlight recommendations for integrating $\mathrm{PT}$ into an interprofessional outpatient HIV care setting. We defined each theme as it related to our study objective and organised the themes to clearly describe participant views and perspectives. ${ }^{29}$ We analysed categorical demographic variables using frequencies and percentages and continuous demographic variables using interquartile ranges (IQRs).

\section{RESULTS}

Twenty-five individuals participated in an interview (12 healthcare professionals) or a focus group (13 adults living with HIV) between January and May 2017. Nine healthcare professionals practised in Canada and three practised in the UK. Half were rehabilitation professionals (three occupational therapists and three physiotherapists) and the others included a nurse, pharmacist, recreation therapist, social worker, physician and massage therapist. All three participants from the UK were rehabilitation professionals. Five healthcare professionals worked in a specialty hospital, five in a hospital and two in 
a community health centre or health clinic. The healthcare professionals reported a median of 12 (IQR 8-16) years of experience working with people living with HIV and a median of 9 years (IQR 4-12) working in a community setting, defined as care provided to people living with HIV outside of a hospital. Table 1 summarises the characteristics of the focus group participants living with HIV. The majority of adults living with HIV were men and self-reported living with a median of 6 (IQR 3-13) concurrent health conditions in addition to HIV. Approximately one-third (four) of participants living with HIV had no access to provincial social assistance or extended health benefits. Nine were current clients of Casey House and 10 reported interest in attending a day health programme for people living with HIV.

We present overall impressions of PT in outpatient HIV care, followed by factors to consider when implementing PT in an interprofessional care setting using the following themes: promoting the role of, and evidence for, PT in outpatient HIV care; structuring the PT mode of delivery to accommodate the unique needs and priorities of people living with HIV; working collaboratively with a physiotherapist on the healthcare team and evaluating rehabilitation as a component of an interprofessional HIV care. We integrated perspectives of both healthcare professionals with expertise in HIV care and people living with HIV to best represent recommendations for implementing PT as part of interprofessional outpatient HIV care. Given the diversity of professions represented in our sample, we refer to healthcare professionals as either 'rehabilitation professionals' (physiotherapist or occupational therapist) or 'other healthcare professionals' (social worker, recreational therapist, pharmacist, physician, registered nurse and massage therapy) in order to maintain participant anonymity.

\section{Impressions of physiotherapy in outpatient HIV care}

Participants living with HIV expressed perceived benefits of having access to PT in an outpatient, interprofessional care setting:

When I walk, I'm not quite as strong as I used to be. I need to be careful when I walk. Physiotherapy, I think, will open up a whole new avenue for me and give me more confidence and actually, walking from

A to B. Person living with HIV—P7 (man)

Another participant described how having quick access to publicly funded PT may be beneficial to those experiencing acute challenges related to self-care, housing or mobility:

I think that might be a good idea, rapid access, someone coming in off the street who is HIV positive having a hard time walking, or, you know, not quite taking care of themselves, that can see someone fairly quickly, talk to them and maybe, you know, get some kind of physiotherapy. Person living with HIV—P1 (man)
Table 1 Participant characteristics: adults living with $\mathrm{HIV}^{\star}$

Number of participants

Characteristic (\%)

\begin{tabular}{lc}
\hline Gender & \\
Man & $11(85)$ \\
Woman & $2(15)$ \\
Median age in years) (IQR) ( $\mathrm{n}=11$ responses) & $50(47-55)$ \\
Current marital status ( $\mathrm{n}=10$ responses) & \\
Single & $8(80)$ \\
Widowed & $2(20)$ \\
$\begin{array}{l}\text { Currently working or volunteering } \\
\text { (n=11 responses) }\end{array}$ & $3(27)$ \\
Self-reported health ( $\mathrm{n}=11$ responses) & \\
Excellent & $2(18)$ \\
Good & $2(18)$ \\
Fair & $5(45$ \\
Poor & $1(9)$
\end{tabular}

Average gross yearly income before tax ( $n=10$ responses)

\begin{tabular}{|c|c|}
\hline$<\$ C 10000$ & $1(10)$ \\
\hline$\$ C 10000$ to $<\$ C 20000$ & $3(30)$ \\
\hline$\$ C 20000$ to $<\$ C 30000$ & $5(50)$ \\
\hline$\$ C 30000$ to $<\$ C 60000$ & $0(0)$ \\
\hline$\$ C 60000$ to $<\$ C 70000$ & $1(1)$ \\
\hline \multicolumn{2}{|l|}{$\begin{array}{l}\text { Extended medical benefits coverage status } \\
\text { ( } n=11 \text { responses) }\end{array}$} \\
\hline $\begin{array}{l}\text { No medical insurance benefits other than } \\
\text { provincial healthcare }\end{array}$ & $4(36)$ \\
\hline $\begin{array}{l}\text { Benefits through a provincial social } \\
\text { assistance plan }\end{array}$ & $3(27)$ \\
\hline $\begin{array}{l}\text { Extended medical insurance coverage } \\
\text { through work }\end{array}$ & $1(9)$ \\
\hline Other† & $3(27)$ \\
\hline $\begin{array}{l}\text { Year of HIV diagnosis, median (IQR) } \\
\text { ( } n=11 \text { responses) }\end{array}$ & $\begin{array}{l}1997(1995- \\
2002)\end{array}$ \\
\hline $\begin{array}{l}\text { Currently taking HIV antiretroviral therapy } \\
\text { ( } n=11 \text { responses) }\end{array}$ & $9(69)$ \\
\hline $\begin{array}{l}\text { Viral load undetectable ( }<50 \text { copies } / \mathrm{mL}) \\
\text { ( } \mathrm{n}=11 \text { responses) }\end{array}$ & $7(64)$ \\
\hline $\begin{array}{l}\text { Number of self-reported concurrent health } \\
\text { conditions in addition to HIV, median, (IQR) } \\
\text { ( } \mathrm{n}=11 \text { responses) }\end{array}$ & $6(3-13)$ \\
\hline \multicolumn{2}{|c|}{$\begin{array}{l}\text { Commonly self-reported concurrent health conditions } \neq \S \\
\text { ( } n=11 \text { responses) }\end{array}$} \\
\hline Muscle pain & $7(64)$ \\
\hline Dental problems & $6(55)$ \\
\hline HIV wasting syndrome & $6(55)$ \\
\hline Joint pain & $6(55)$ \\
\hline Mental health condition & $6(55)$ \\
\hline
\end{tabular}

Continued 


\section{Table 1 Continued}

\begin{tabular}{ll} 
Characteristic & (\%) \\
\hline $\begin{array}{l}\text { Experience with physiotherapy } \\
\text { (n=11 responses) }\end{array}$ & $3(27)$ \\
$\quad$ Currently seeing a physiotherapist & $6(54)$ \\
Saw a physiotherapist in the past year & $2(18)$ \\
$\quad$ Never saw a physiotherapist & $8(73)$ \\
$\begin{array}{l}\text { Commonly reported reasons for seeing physiotherapistł§ } \\
\text { (n=11 responses) }\end{array}$ & $7(64)$ \\
$\begin{array}{l}\text { To address HIV and side effects of } \\
\text { treatment }\end{array}$ & $\begin{array}{l}\text { To address issues related to other health } \\
\text { conditions }\end{array}$ \\
$\begin{array}{l}\text { To address physical health challenges } \\
\text { To address challenges carrying out day-to- }\end{array}$ & $6(55)$ \\
day activities & \\
$\begin{array}{l}\text { To help get back to leisure or recreational } \\
\text { activities }\end{array}$ & $5(45)$
\end{tabular}

*11 of 13 adults living with HIV completed the demographic questionnaire.

†Other funding included status card (government ID card for which some Indigenous peoples are eligible, and which provides some extended health coverage) and unspecified.

$\ddagger$ Reported by $\geq 5$ participants.

§Participants were asked to check all response options that apply.

Another effective use of time clinically... could be that ... we're going out to the homeless and we're giving them (clients living with HIV) mobility aids and then you could get them in (to PT) because then they know it's (the physiotherapy program) free and they can access something. Rehabilitation professional-P10 (UK)

One participant spoke about his challenges accessing PT in the past, attributed to having to pay out of pocket for services. He described how universal access to PT as part of a publicly funded, outpatient day health programme could facilitate access to rehabilitation for more complex and marginalised populations with limited income and financial insecurity:

I just didn't follow it (physiotherapy) through because of the problem with paying and getting reimbursed. But if there was something like the day programme and I could have accessed one appointment every 2 or 3 weeks I would have probably tended to the problem (Baker's cyst in the knee). The way it was I didn't do anything about it. Person living with HIV—P6 (man)

Healthcare professionals described how an outpatient interprofessional approach to care offered the potential to 'pull in those people who are reluctant to engage elsewhere' and 'fill a big gap in the clinical and psychosocial care of our patients'. One rehabilitation professional discussed the value of a specialised outpatient form of care in this population:

Why can't they (people living with HIV) access a musculoskeletal outpatient service or neuro outpatient service or general sort of physio clinics?... for some people living with HIV, where their disease is well controlled, they're not having social problems, mental health problems, that may well be true... but there's a fairly big proportion of people, or certainly a reasonable community of people living with HIV who have complex care morbidities and I think it's those people that really need... special services. Rehabilitation professional-P11 (UK)

Healthcare professionals suggested that an interprofessional approach to care in the outpatient setting has potential to address gaps in the healthcare system by incorporating programmes and services, such as PT that are non-existent or are inaccessible to people living with HIV:

Once you're out the door in our healthcare system, you're on your own. So the more guidance we give them (people living with HIV), the more education, the better. With our patients, a lot of issues come up because of their cognitive impairment, so even if they're told some things, they need constant reminders about how to take care of themselves. Other healthcare professional-P3 (Canada)

\section{Factors to consider when implementing physiotherapy in outpatient interprofessional HIV care}

In order to adequately address the complexity of HIV care in a practical setting, we identified four themes regarding the implementation of PT into outpatient interprofessional HIV care: (1) promoting the role of, and evidence for, PT in an outpatient HIV clinical setting; (2) structuring the PT mode of delivery to accommodate the unique needs and priorities of people living with HIV; (3) working collaboratively with a physiotherapist on the healthcare team and (4) evaluating rehabilitation as a component of interprofessional care.

Promoting the role of, and evidence for, physiotherapy in an outpatient HIV clinical setting

\section{Role of physiotherapy}

Participants described the role of PT within outpatient HIV clinical settings as addressing physical, psychological and social aspects of health within the context of a health promotion and rehabilitation approach to care. Many participants living with HIV viewed the role of PT as synonymous with exercise, stating that PT in an outpatient interprofessional care setting would provide an opportunity to 'get help with exercises' and engage in 'exercise together (with peers) or go walking together'. In addition, people living with HIV suggested PT could enhance social engagement and provide a venue to build the strength and functional ability to 'actually get up and begin to return to going to a theatre'.

Healthcare professionals similarly referred to the role of PT within an outpatient interprofessional service in a physical 
context such as 'cardiorespiratory, progressive resistance training, neuromotor exercises' and 'balance... falls prevention', as well as psychological and social aspects including 'motivation, inspiration, structure, meaning' while using a creative approach to 'find an activity that actually motivates someone'. Healthcare professionals also viewed physiotherapists as having a role in 'education' and 'preventative health' such as 'falls prevention', and preventing 'secondary complications' and 'pain'.

\section{Promoting physiotherapy in an interprofessional outpatient HIV care setting}

Community-based and hospital-based health professionals noted the importance of information sharing between HIV clinics in the city and an outpatient service (day health programme). They suggested that it was valuable for physiotherapists to visit clinics and to present at rounds in order to inform the healthcare community about the role and evidence in addressing disability and promoting healthy ageing with HIV, and provide practical information about how clients can access services:

Every HIV clinic actually has some kind of rounds. A good way of promoting is to... offer just to do a rounds either what is being offered at (Name of site) or on a topic, on a physio-related topic so what's new in treating or what's new in arthritis and HIV... you get to educate somebody but also are plugging the services at the same time. Other healthcare professional-P6 (Canada)

Another healthcare professional participant, with experience working in an $\mathrm{HIV}$-specific health centre, discussed using social media, specifically involving the experiences of clients to raise awareness of PT and provide practical information about what PT is, and how services available can be accessed in an outpatient clinical setting:

Always use social media... have a Twitter account, have a Facebook page, have a YouTube video of what physiotherapy at (Name of site) is. Those sort of things are important because then if you've got a really engaging video that has a physiotherapist and a service user in it saying this is what physiotherapy is, this is the services we offer, this is what happens when you come and then someone giving their personal experience of attending, that will make the world of difference. Rehabilitation professional-P10 (UK)

\section{Staffing and support}

Participants noted the role members of the healthcare team play in creating a welcoming environment. Healthcare professionals indicated personal traits that would be useful for physiotherapists to possess in order to facilitate engagement in PT such as 'warmth', 'adaptable', 'non-judgemental' and possessing 'broad knowledge (of cardiovascular, neurological and musculoskeletal physical therapy-related specialties and rehabilitation for people living with HIV and marginalised populations)'.
People living with HIV and healthcare professionals explained the importance of having a physiotherapist who has experience in HIV care, and understands the physical, social and psychological complexities of living with HIV.

I saw a physiotherapist, and ... she didn't understand HIV, which is fine, ...she was like, 'oh I've never seen somebody so young be so weak, I usually work with senior citizens', and just made me really feel like an alien, that it was like, 'I don't even want to work with her anymore.' And so that's why I'm kind of like, with something with (a specialty hospital) you feel like people already understand HIV, you don't feel like you have to give a lesson. Person living with HIV—P3 (woman)

However, some participants did not feel all PT services offered to people living with HIV needed to be HIV-specific in nature. Some suggested partnering with other community health and social service-focused programmes, which are not HIV-specific to provide additional options for adults with HIV to address their episodic disability, for issues related to ageing, and disability attributed to potential multimorbidity, such mental health or chronic pain:

I think it's important that there is available knowledge on what other services can be referred to because not everybody wants to come to a HIV specific service. Just because you're positive doesn't mean you have to engage in a positive programme. Rehabilitation professional-P10 (UK)

People living with HIV participants suggested involving PT students in an outpatient care setting such as the day health programme. Integrating PT students offered a cost-effective strategy for increasing availability of PT services while promoting opportunities for increasing knowledge and awareness about HIV and reducing stigma among future healthcare professionals.

It would be cheaper to have students to come as part of their programme or schooling... I think it would help open up the door to, uh, people who are afraid of communicating with HIV/AIDS... There is still stigma about HIV out there. I can only imagine what it is like, going to school, thinking 'oh god, I am going to work with HIV clients, I don't want to touch them, that sort of thing. But, get rid of the fear, educate yourself. Education is key-and this would be part of education. Person living with HIV—P1 (man)

Structuring the physiotherapy mode of delivery to accommodate the unique needs and priorities of people living with HIV

\section{Client-oriented environment}

Participants emphasised the importance of a 'welcoming' environment including 'bright and cheery colours' to make it 'as much of a comfortable experience' as possible. One healthcare professional noted that people with HIV may be more likely to access PT in an outpatient 
interprofessional setting knowing that 'they can access more than one thing that's free' in one location. Participants suggested reminder phone calls can be beneficial in promoting attendance for outpatient service appointments amid fluctuating health, various medical appointments and scheduling:

I think that (an) appointment reminder is crucial for people like us who are inundated with appointments. Person living with HIV—P6 (man)

\section{Group versus individual sessions}

Both healthcare professionals and people with HIV expressed the benefits of group PT exercise and education sessions including peer support, motivation and cognition:

Peer-engaged support, you pair people up, ... we get to know each other, and you don't create dependent links that emerge as you're doing physio... pair people, encourage people, because then you forget a part of an exercise, and then my... peer remembers the rest of it. Person living with HIV-P12 (man)

However, both stakeholder groups also acknowledged the need for individualised PT sessions, specifically for initial assessments prior to joining a group, to ensure the unique needs of a given client are met:

One-on-one is really good if the client has really specific goals around walking or safety or improving transfers... Group settings again have potential for group teaching or exercise class and also has that opportunity to bring folks together and feel like a community. Other healthcare professional-P5 (Canada)

\section{Structured versus flexible approaches to physiotherapy care}

Participants recommended that scheduled PT appointments are important, but that a PT service should be flexible in order to facilitate access to people who may experience episodic disability and other confounding barriers to attending PT:

I think that having flexibility allows for options and allows for choices because living with HIV you can have 1 day that's great and the next is not so great because the condition is episodic in nature. So it's an episodic disability just like cancer, lupus, arthritis, MS that sort of thing. Even within the day you can be great in the morning by 10 o'clock and then by 1 o'clock you're not feeling that great. Rehabilitation professional-P7 (Canada)

One healthcare professional described the benefits of a group-based approach offering a flexible (drop-in) attendance schedule in his work setting:

We now have open access, which is, we have people attend, return or restart depending on their own health and disability. So the open accessibility almost enables people to take a bit more ownership over their health and they can engage in these things a bit more. They create a little bit more autonomy about what is important for them in a supervised, safe physiotherapy led environment. Rehabilitation professional-P10 (UK)

\section{Client-oriented goals and interventions}

Participants expressed the need to engage in meaningful PT programmes that are relevant and tailored to clients' goals, abilities and preferences within the context of their day-to-day lives. One person living with HIV indicated the importance of PT to address functional goals specific to the individual in order to have a relevant and meaningful impact on daily living:

It's different when you're in a controlled setting like that (clinical), as opposed to walking the street on your own, so it's sort of like a clinical versus a day-today therapy. So even things like walking the sidewalks and learning how to not trip over things or learning to go up your stairs. Person living with HIV—P5 (man)

One healthcare professional with experience working in an HIV outpatient setting emphasised the importance of an intervention-focused approach tailored specifically to clients' goals:

When we're addressing what's meaningful and important to the individual that we're treating, if they engage with the process and engage with physical therapy or physiotherapy, we can achieve people's goals and we know that the majority of their goals are either body image concerns, participation in meaningful tasks, health and fitness or mobility. Rehabilitation professional-P10 (UK)

One participant with HIV noted how participating in PT could allow individuals to feel a sense of purpose in contributing to community:

...they (people living with HIV) get involved in the community and I know that there's people at this table that are working at the food bank, and the physiotherapy can give them energy and extra strength and so with the physiotherapy... you are able to give back to the community and I think that's wonderful. Person living with HIV_P12 (man)

In order to address clients' individual goals and unique presentations, healthcare professionals suggested stratifying interventions. Practically, this could involve 'different groups for people at different levels' and a varying 'ratio' of participants to support staff depending on factors such as 'cognitive problems', 'comorbidities', 'age' and 'mood issues'. One rehabilitation professional participant noted:

We need to make sure that what we're doing is... centred on the individual, so I think that everybody who you see, you should do a thorough assessment and 
kind of work out what their needs are. Rehabilitation professional-P12 (UK)

One participant living with HIV described his concerns regarding a group-based intervention that was not tailored to his level of ability:

If I'm in a group setting and they're doing something that I find a bit difficult, I (want to be) able to go to a one on one and learn how to do it without taking away time from everyone else and learning for myself so I'm... secure enough in myself to know I can do the move without toppling over or bothering something. Person living with HIV—P2 (man)

\section{Promoting independence}

One participant living with HIV identified that it can be 'very hard doing physiotherapy without (a physiotherapist) in your room, because she'll come and make sure you exercise'. Another participant suggested how to overcome this challenge:

The knee exercises you are doing in the studio or in the centre... record it for each participant so that each participant has their own disc to take home and follow-through because three days a week. Three days off, four days on. There are your instructions there. Person living with HIV—P13 (man)

Health professionals described how PT resources and materials should be adapted to maximise retention, independence and adherence to PT programmes:

I think that ensuring if you are using any print material that you are using basic language... Easy to access information. If material is in print, can our clients read it?... Understanding that people might need to have the same session two or three times to retain that session. Other healthcare professional-P5 (Canada)

\section{Sensitivity to practice}

People with HIV and healthcare professionals noted the importance of physiotherapists to adopt approaches that are sensitive to the complexities sometimes faced by people with HIV including the potential episodic nature of HIV, stigma, substance use challenges and financial insecurity. One participant emphasised the importance of a 'safe space' to address stigma with HIV.

I think that one of the key things is that providing physiotherapy in a safe space... which is a space which is maybe dedicated and specialised to people living with HIV... I think is incredibly important for some people. I think some people want the opportunity to know that even though they don't have to talk about HIV, if I want to talk about HIV in the context of why I'm here, I'm not going to be judged, I'm not going to be stigmatised against... I'm not going to encounter something negative. Rehabilitation professional-P10 (UK)
Participants reported stigma as a barrier to accessing PT and suggested the first step in mitigating stigma is to simply acknowledge its presence. Stigma may be related to various aspects of life, including, 'mental health', 'homosexuality', 'HIV status' and may come from health professionals, family or internalised stigma. Participants suggested offering group exercise classes tailored to individuals who may identify with a certain culture or gender while considering sensitivity to PT practice in order reduce the potential for stigma and discrimination:

Certain cultures, men and women in the same room... partnering up and things like that... also gender... the trans community as well... so it would be engaging them as well. Person living with HIV—P6 (man)

People living with HIV and healthcare professionals recognised substance use, including 'alcohol' and 'drug use' specifically, 'cigarettes', 'crystal meth' and 'cocaine', as a barrier to participation in PT:

So we try to schedule the appointments... but sometimes they are not (adherent) because unfortunately the drug use or the alcohol, whatever they are using, the substance use is a stronger pull. I would say very often if they have true addiction issues, then it can ... interfere very much. Rehabilitation professional-P8 (Canada)

One person with HIV suggested 'a harm reduction framework within the physiotherapy' approach to better address needs of clients. Healthcare professionals noted concerns regarding the associated risk allowing clients who are using substances to participate in PT due to impaired balance, judgement and potentially unstable vitals: "I certainly didn't feel safe to bring people in (to physiotherapy) who are on substances".

Working collaboratively with a physiotherapist on the healthcare team

\section{Team communication}

Participants recognised the importance of communication to streamline referrals and to discuss progress of clients within the team. One rehabilitation professional who works in an interprofessional setting discussed the importance of identifying appropriate referrals to PT:

They (clients) need to be identified as requiring physiotherapy...what is the criteria of requiring physiotherapy...the triggers for referral, what is the threshold for referral and ... the appropriate pathway to facilitate engagement and accessibility? Rehabilitation professional-P10 (UK)

The healthcare team should be knowledgeable of the other members on the team and services available to provide client-centred care. Some healthcare professionals suggested regular meetings in which the team can discuss concerns and specific clients who may be attending the programme that day. 
I think communication is the biggest thing, so if you can build tools upfront like weekly meetings or even daily meetings... focusing on specific pieces of, like clinical issues that are coming up then, you're probably going to have more success in providing patient care to people. Other healthcare professional-P4 (Canada)

Some health professionals suggested that PT may have a role in informing other health practices in regard to transfers, pain, physical impairments and mobility for clients. One healthcare professional who worked in an interprofessional setting described how the team can reinforce PT recommendations so that clients can be best supported, using personalised strategies and techniques in each environment:

They (physiotherapists) could inform the work that I do, and... it would probably inform what massage therapy does as well and what nursing does, it already informs what nursing does, but I think more heavily...nursing-our nurses are great at implementing the recommendations of physio. Other healthcare professional-P2 (Canada)

\section{Interprofessional group sessions}

Some health professionals suggested adopting interprofessional sessions involving a physiotherapist and another health professional, while others advised against it. One rehabilitation professional commented on the challenge of addressing competing priorities in a joint session:

When we have more than one professional in the clinic room at the time, completely ineffectual. I did a joint clinic with a dietician, didn't work. There's too many people in the room, too many factors to consider, too many competing issues for prioritising what's important at the time. Rehabilitation professionalP10 (UK)

However, another health professional commented on the potential benefits of interprofessional collaboration in a shared group programme:

I would love to see a collaboration between physiotherapy and recreation therapy around some sort of exercise groups in the future within the day health programme. That would be something that I would-I think would be a really natural pairing and would work really well. Other healthcare professional-P2 (Canada)

Whether encouraging interprofessional group-based sessions or not, both healthcare professionals and adults living with HIV recognised the importance of identifying common goals to facilitate an effective and meaningful PT session.

Evaluating rehabilitation as a component of interprofessional care Many healthcare professionals discussed the importance of evaluation to determine the successes and challenges of implementing a new discipline such as PT in an interprofessional, outpatient HIV care setting. The episodic nature of HIV, in addition to the complex physical, psychological and social domains of health affected, requires a broad approach to programme evaluation. One health professional with many years experience in HIV care reported:

It's very difficult to find a uniform measurement tool to look at objective markers of success with physical therapy in a heterogeneous population such as ...people with HIV. Which is the problem we face, which is why the subjective tools and... measurements are important. However, measuring success means measuring change over time and I think that when you are looking at a condition that is episodic... I think it's important that we look at a range of different things. So I think there needs to be a battery approach. Rehabilitation professional-P10 (UK)

Most healthcare professionals suggested implementing a variety of evaluation methods, focused on client goals to capture subjective and objective components of evaluation.

I think all evaluation needs to consider what the patient goals are, so to be less weighted around programme goals and maybe being more focused around patient goals that might be one way to consider the evaluation. Other healthcare professional- $\mathrm{P} 4$ (Canada)

One participant with HIV suggested that evaluating PT as a component of interprofessional HIV care should involve "weekly or monthly check-ins... just a couple of simple questions' for people with HIV to answer. Another suggested:

Once you start getting clients, like the ones that are seeing the physiotherapist, ask them how its working and how they think it is going so you guys could know how everybody is doing with it. Person living with HIV—P4 (woman)

Overall, participants recognised the importance of focusing on the clients' goals and perspectives to effectively and rigorously evaluate rehabilitation (and specifically PT) as a component of interprofessional HIV care.

\section{DISCUSSION}

To our knowledge, this is the first study to explore factors to consider when implementing PT into an outpatient interprofessional HIV care setting. The role of PT in HIV care is multidimensional and client-centred. Our results recommend a goal-oriented and person-centred PT approach to care. Our findings align with recommendations outlined in a conceptual framework of rehabilitation for people living with $\mathrm{HIV}^{6}$ and highlight the need for rehabilitation in outpatient settings to address 
prevention and healthy ageing concerns such as mobility and social engagement for people with HIV.

Our results indicate the importance of evaluating PT as an interprofessional approach to outpatient HIV care. In the evaluation of a physiotherapist-led group outpatient rehabilitation programme for people living with HIV in the UK, individualised goal-setting was beneficial, as $83 \%$ of participants achieved or surpassed their goals. ${ }^{11}$ Client-centred HIV care should allow for flexibility to accommodate the potential episodic nature of HIV. Participants outlined barriers to accessing and engaging in PT, which stemmed from the chaotic lifestyle some people might experience related to substance use, stigma, financial security and basic needs (housing and food) in addition to the episodic nature of HIV. Brown et al minimised these barriers by designing a PT programme where participants were not required to attend weekly, but free to attend and restart as able. ${ }^{11}$ Collectively our findings highlight the evolving role of rehabilitation beyond tertiary care to that of primary and preventative care as a mechanism for health promotion, prevention of multimorbidity and healthy ageing with HIV.

Results from our study highlight the importance of a physiotherapist working in an outpatient setting to maintain communication with community HIV clinics ensuring health providers know what services are offered and how to refer clients to PT. Chetty and Hanass-Hancock conceptualised a rehabilitation model of care for people living with HIV in South Africa. ${ }^{30}$ Authors highlighted the importance of communication among multidisciplinary team members and between hospital and community settings in order to optimise rehabilitation and the need for physiotherapists to provide ongoing education with team members and clients regarding the role and importance of PT. ${ }^{30}$ Studies with other chronic disease populations including chronic heart failure and diabetes have shown that lack of interprofessional communication is a barrier to providing optimal care. ${ }^{31}{ }^{32}$ Authors of a qualitative descriptive study explored the impact of introducing an initiative to increase interprofessional communication among healthcare professionals working with patients with heart failure. After introducing strategies to enhance communication, professionals felt they had greater knowledge of heart failure, and felt patients had improved clinical outcomes. ${ }^{33}$ While the importance of interprofessional communication within an outpatient service is evident, further research is needed to address how to optimise communication along the healthcare continuum, particularly with episodic illness where the continuum may not always be predictable, nor linear in nature.

With HIV having transitioned from a progressively terminal to a chronic and episodic illness, the rehabilitation needs of people now ageing with HIV are increasing, and shifting from the traditional inpatient (hospital) setting to the outpatient (day health programme or community) setting. However, few people with HIV are accessing formalised PT services citing barriers related to financial constraints, physical barriers and lack of knowledge and awareness among members of the healthcare team about the role for rehabilitation. ${ }^{30} 34$

Participants in this study noted the importance of physiotherapists to connect with non-HIV-specific intervention locales such as community-based PT and fitness centres in order to facilitate referrals if clients prefer to seek treatment in a non-HIV-specific setting. In a study examining factors to consider when developing a community-based exercise programme for people with HIV, some participants with HIV preferred to attend a programme which was not HIV specific. Some felt people with HIV had similar PT priorities as the general population and could attend any programme, while others stated attending an HIV programme meant exposing themselves to the potential stigma associated with HIV disclosure..$^{35}$ A qualitative synthesis highlighted experiences of stigma within an HIV care setting, including segregation of people with HIV, behaviours of healthcare professionals related to fears of exposure and perceived judgement from practitioners. ${ }^{36}$ Fear of stigma attending a general outpatient clinical setting was evident among some participants in our study who expressed preferences for an HIV-specific programme due to beliefs that health providers would better understand and be able to address their needs. Participants echoed benefits of social support, such as group PT sessions and showed potential benefits of mitigating stigma by involving PT students in an HIV-specific outpatient service. Education on the role and evidence of PT in HIV care is critical for enhancing awareness among current and emerging health professionals about $\mathrm{PT}^{37}$ and can help to mitigate stigma. While our study provides some insight, further research is necessary to determine how best to provide accessible care for those experiencing HIV-related stigma.

Participants in this study were living with a median of six concurrent health conditions with the majority reporting mental health concerns. Participants commented on challenges accessing healthcare services for those who are living with HIV and actively using drugs and alcohol. These principles are reflective of specific considerations related to sensitive practice when implementing PT assessment and treatment in an outpatient care setting. Interprofessional online modules which address some of these topics, demonstrated utility for increasing education and awareness of rehabilitation for people living with HIV among community organisations, people with HIV as well as current and future healthcare professionals. ${ }^{20} 3839$

Limited evidence exists concerning integration of PT into interprofessional outpatient care settings for populations with chronic health conditions. While models of care were developed to better meet the healthcare needs of populations living with chronic conditions, specifically in underserviced areas, ${ }^{40}{ }^{41}$ few involve PT and few are specific to HIV care. Chetty and Hanass-Hancock developed a rehabilitation model of care for people living with HIV in South Africa. ${ }^{30} 42$ Authors concluded the need for a patient-centred and multidisciplinary approach to care, 
similarly reported by participants in our study. Guiding principles for a rehabilitation model of care included effective communication, leadership, collaboration and education of providers in order to successfully implement rehabilitation across home and community-based care settings. ${ }^{30}$ There is an opportunity for the HIV and rehabilitation sector to learn and apply evidence from other illnesses such as chronic obstructive pulmonary disease ${ }^{43}$ and fibromyalgia, ${ }^{44}$ and in older adults living with multimorbidity, ${ }^{45}$ as well as applying principles from models of HIV rehabilitation established in other contexts such as low-income to middle-income countries. ${ }^{30} 42$ As more people age with HIV in combination with other chronic conditions, it will be important to draw from evidence on the effect of PT interventions from other chronic conditions such as chronic obstructive pulmonary disease, ${ }^{46}$ diabetes ${ }^{47}$ and osteoporosis, ${ }^{48}$ and apply it to the context of people with HIV in the outpatient care setting.

In this study, we used a newly emerging day health programme in Toronto, Canada as an exemplar to establish recommendations for integrating PT into an interprofessional outpatient HIV care setting. We interviewed a variety of healthcare professionals from Canada and the UK to gain a broad range of perspectives. Although few health professionals had experience with community-based HIV care, all had experience working in HIV patient care and as such were capable of speaking to the potential role of PT. Our aim was not to achieve saturation, but rather to obtain a rich description of perspectives related to HIV PT care. Nevertheless, we ceased data collection with 25 participants, which we observed as the point when no new categories emerged. As the recruitment for people living with HIV was done through an HIV-specialty hospital in Toronto, our study population consisted of adults living with a median of six comorbid conditions in addition to HIV and many who had accessed PT in the past year. Results may not represent those living in rural areas or low-to middle-income countries who may have distinct barriers to healthcare resources. Nevertheless, the considerations from our study related to the importance of client-centred, goal-oriented and interprofessional care is analogous to the guiding principles of a rehabilitation model of care for people living with HIV in South Africa. ${ }^{30}$ Further research is necessary to investigate the potential for cost-saving, client-centred interprofessional approaches to care which may be relevant across different health systems and settings.

\section{CONCLUSION}

Factors for consideration when implementing PT into an interprofessional outpatient HIV care setting include: promoting the role of, and evidence for, incorporating PT in outpatient HIV care, structuring PT delivery to accommodate the unique needs and priorities of people living with HIV, working collaboratively with a physiotherapist on the healthcare team, and evaluating rehabilitation as a component of interprofessional care. Results may be used by people living with HIV, clinicians (health and rehabilitation professionals), administrators and policy stakeholders to inform the planning and integration of timely and appropriate access to evidence-informed rehabilitation into interprofessional care for people living with chronic illness, such as HIV.

Acknowledgements We completed this research in partial fulfillment of the MScPT degree requirements at the University of Toronto. The authors would like to thank the participants of this study, including the people living with HIV and healthcare professionals. The authors would like to acknowledge the contributions of Sarah Munce, Ken King, as well as Giovanni lacono and other Casey House staff in protocol development, recruitment and data collection.

Contributors KKOB (PhD) and SCC (PhD) designed the study and provided guidance throughout the research process. KKOB and SCC possess expertise in qualitative methodology and HIV and exercise research. KKOB and SCC supervised $\mathrm{HdB}, \mathrm{SC}, \mathrm{MA}, \mathrm{EL}$ and AP (MScPT students) who developed the protocol, collected and analysed the data, and drafted the manuscript in partial fulfillment of requirements for an MScPT degree at the University of Toronto. HdB, SC, MA, EL and AP (MScPT students) developed skills in qualitative research methodology including attending lectures; completing readings on qualitative research study design; understanding steps of recruitment, data collection and analysis; completing a literature review; developing the research protocol, interview guides, focus group guide and demographic questionnaire and considering the ethical issues associated with this research. All steps were closely reviewed and guided by KKOB and SCC (advisors). All authors read and approved the final manuscript.

Funding This research was partially funded by an Ontario Physiotherapy Association Central Toronto District of Toronto MScPT Research Grant Award. Kelly K. O'Brien was supported by a New Investigator Award from the Canadian Institutes of Health Research (ClHR) and an Early Researcher Award with the Ontario Ministry of Research and Innovation. Kelly K. O'Brien is supported by a Canada Research Chair (CRC) in Episodic Disability and Rehabilitation. This research was undertaken, in part, thanks to funding from the Canada Research Chairs program.

Competing interests None declared.

Patient consent for publication Obtained.

Ethics approval The study was approved by the University of Toronto HIV/AIDS Research Ethics Board (Protocol Reference \#33760).

Provenance and peer review Not commissioned; externally peer reviewed.

Data sharing statement The data collected and analysed during the study are not publicly available in accordance with our study protocol that was approved by the University of Toronto HIV/AIDS Research Ethics Board. Data may be available on reasonable request by contacting the corresponding author.

Open access This is an open access article distributed in accordance with the Creative Commons Attribution Non Commercial (CC BY-NC 4.0) license, which permits others to distribute, remix, adapt, build upon this work non-commercially, and license their derivative works on different terms, provided the original work is properly cited, appropriate credit is given, any changes made indicated, and the use is non-commercial. See: http://creativecommons.org/licenses/by-nc/4.0/.

\section{REFERENCES}

1. Johs NA, Wu K, Tassiopoulos K, et al. Disability among middle-aged and older persons with HIV infection. Clin Infect Dis 2017;65:83-91.

2. Deeks SG, Lewin SR, Havlir DV. The end of AIDS: HIV infection as a chronic disease. Lancet 2013;382:1525-33.

3. Antiretroviral Therapy Cohort Collaboration. Survival of HIV-positive patients starting antiretroviral therapy between 1996 and 2013: a collaborative analysis of cohort studies. Lancet HIV 2017;4:e349-56.

4. Kendall CE, Wong J, Taljaard M, et al. A cross-sectional, populationbased study measuring comorbidity among people living with HIV in Ontario. BMC Public Health 2014;14:161.

5. O'Brien KK, Bayoumi AM, Strike C, et al. Exploring disability from the perspective of adults living with HIV/AIDS: development of a conceptual framework. Health Qual Life Outcomes 2008;6:76.

6. Worthington C, Myers T, O'Brien K, et al. Rehabilitation in HIV/AIDS: development of an expanded conceptual framework. AIDS Patient Care STDS 2005;19:258-71. 
7. Cobbing S, Hanass-Hancock J, Deane M. Physiotherapy rehabilitation in the context of HIV and disability in KwaZulu-Natal, South Africa. Disabil Rehabil 2014;36:1687-94.

8. Pullen S. Physical therapy as non-pharmacological chronic pain management of adults living with HIV: self-reported pain scores and analgesic use. Hiv Aids 2017;9:177-82.

9. Kietrys DM, Galantino ML, Cohen ET, et al. Yoga for persons with hiv-related distal sensory polyneuropathy. Rehabil Oncol 2018;36:123-31.

10. O'Brien KK, Solomon P, Trentham B, et al. Evidence-informed recommendations for rehabilitation with older adults living with HIV: a knowledge synthesis. BMJ Open 2014;4:e004692.

11. Brown D, Claffey A, Harding R. Evaluation of a physiotherapy-led group rehabilitation intervention for adults living with HIV: referrals, adherence and outcomes. AIDS Care 2016;28:1495-505.

12. O'Brien KK, Tynan AM, Nixon SA, et al. Effectiveness of aerobic exercise for adults living with HIV: systematic review and metaanalysis using the Cochrane Collaboration protocol. BMC Infect Dis 2016;16:182.

13. O'Brien KK, Tynan AM, Nixon SA, et al. Effectiveness of Progressive Resistive Exercise (PRE) in the context of HIV: systematic review and meta-analysis using the Cochrane Collaboration protocol. BMC Infect Dis 2017:17:268.

14. Worthington $\mathrm{C}$, Myers $\mathrm{T}$, O'Brien $\mathrm{K}$, et al. Rehabilitation professionals and human immunodeficiency virus care: results of a national Canadian survey. Arch Phys Med Rehabil 2008:89:105-13.

15. Worthington C, O'Brien K, Myers T, et al. Expanding the lens of HIV services provision in Canada: results of a national survey of HIV health professionals. AIDS Care 2009;21:1371-80.

16. Cobbing S, Hanass-Hancock J, Myezwa H. A home-based rehabilitation intervention for adults living with HIV: a randomized controlled trial. J Assoc Nurses AIDS Care 2017;28:105-17.

17. Gough K, Karapita S. Facing the future together: an innovative response to the urgent HIVIAIDS crisis in Toronto. Toronto (ON: Casey House, 2011

18. deBoer $\mathrm{H}$, Andrews $\mathrm{M}$, Cudd $\mathrm{S}$, et al. Where and how does physical therapy fit? Integrating physical therapy into interprofessional HIV care. Disabil Rehabil 2018:10:1-10.

19. O'Brien KK, Wilkins A, Zack E, et al. Developing clinical practice guidelines in HIV rehabilitation: process recommendations and guiding principles. AIDS Educ Prev 2011;23:457-68.

20. Solomon P, Salvatori P, Guenter D. An interprofessional problembased learning course on rehabilitation issues in HIV. Med Teach 2003;25:408-13.

21. Solomon P, O'Brien K, Hard J, et al. An HIV mentorship programme for rehabilitation professionals: lessons learned from a pilot initiative. Int J Ther Rehabil 2011;18:280-9.

22. Sandelowski M. What's in a name? Qualitative description revisited. Research in nursing \& health 2010;33:77-84.

23. Casey House. Casey House. Day Health Program. https://www. caseyhouse.com/how-we-help/day-health-program-services/ (Accessed 5 Mar 2019).

24. O'Brien KK, Solomon P, Ibáñez-Carrasco F, et al. Evolution of an International Research Collaborative in HIV and Rehabilitation: Community Engaged Process, Lessons Learned, and Recommendations. Prog Community Health Partnersh 2018;12:395-408.

25. O'Brien KK, Ibáñez-Carrasco F, Solomon P, et al. Advancing research and practice in HIV and rehabilitation: a framework of research priorities in HIV, disability and rehabilitation. BMC Infect Dis 2014:14:3851.

26. Braun V, Clarke V. Using thematic analysis in psychology. Qual Res Psychol 2006;3:77-101.

27. Hsieh HF, Shannon SE. Three approaches to qualitative content analysis. Qual Health Res 2005;15:1277-88.
28. NVivo qualitative data analysis software. Version 10. [program]. 2012.

29. Sandelowski M. What's in a name? Qualitative description revisited. Res Nurs Health 2010;33:77-84.

30. Chetty V, Hanass-Hancock J. A rehabilitation model as key to comprehensive care in the era of HIV as a chronic disease in South Africa. AIDS Care 2016;28(Suppl 1):132-9.

31. Strachan $\mathrm{PH}$, Kaasalainen $\mathrm{S}$, Horton $\mathrm{A}$, et al. Managing heart failure in the long-term care setting: nurses' experiences in Ontario, Canada. Nurs Res 2014;63:357-65.

32. Raaijmakers LG, Hamers FJ, Martens MK, et al. Perceived facilitators and barriers in diabetes care: a qualitative study among health care professionals in the Netherlands. BMC Fam Pract 2013;14:114.

33. Boscart VM, Heckman GA, Huson K, et al. Implementation of an interprofessional communication and collaboration intervention to improve care capacity for heart failure management in long-term care. J Interprof Care 2017;31:583-92.

34. Chetty V, Hanass-Hancock J, Myezwa H. Expert consensus on the rehabilitation framework guiding a model of care for people living with HIV in a South African Setting. J Assoc Nurses AIDS Care 2016;27:77-88

35. Li A, McCabe T, Silverstein E, et al. Community-based exercise in the context of HIV: factors to consider when developing and implementing community-based exercise programs for people living with HIV. J Int Assoc Provid AIDS Care 2017;16:267-75.

36. Chambers LA, Rueda S, Baker DN, et al. Stigma, HIV and health: a qualitative synthesis. BMC Public Health 2015;15:848.

37. Cobbing S, Chetty V, Hanass-Hancock J, et al. The essential role of physiotherapists in providing rehabilitation services to people living with HIV in South Africa. South African Journal of Physiotherapy 2017;69:22-5.

38. Solomon P, Guenter D, Salvatori P. Integration of persons with HIV in a problem-based tutorial: a qualitative study. Teach Learn Med 2003;15:257-61.

39. Solomon P, Salbach N, O'Brien K, et al. Increasing capacity in rehabilitation in the management of hiv: a case-based email intervention. Journal of Continuing Education and Professional Development 2015;2:1-8.

40. Frakes KA, Brownie S, Davies $L$, et al. Experiences from an interprofessional student-assisted chronic disease clinic. $J$ Interprof Care 2014:28:573-5.

41. Iddins BW, Frank JS, Kannar P, et al. Evaluation of team-based care in an urban free clinic setting. Nurs Adm Q 2015;39:254-62.

42. Chetty V, Hanass-Hancock J. The need for a rehabilitation model to address the disparities of public healthcare for people living with HIV in South Africa. Afr J Disabil 2015;4:137.

43. Peytremann-Bridevaux I, Staeger P, Bridevaux PO, et al. Effectiveness of chronic obstructive pulmonary disease-management programs: systematic review and meta-analysis. Am J Med 2008;121:433-43.

44. Jones KD, Bennett RM, Ward RL, et al. Description of a half-day interprofessional fibromyalgia clinic with an evaluation of patient satisfaction. Am J Phys Med Rehabil 2011;90:825-33.

45. Hansen KT, McDonald C, O'Hara S, et al. A formative evaluation of a nurse practitioner-led interprofessional geriatric outpatient clinic. $J$ Interprof Care 2017;31:546-9.

46. Torres-Sánchez I, Valenza MC, Cebriá I Iranzo MDÀ, et al. Effects of different physical therapy programs on perceived health status in acute exacerbation of chronic obstructive pulmonary disease patients: a randomized clinical trial. Disabil Rehabil 2018;40:2025-31.

47. Cade WT. Diabetes-related microvascular and macrovascular diseases in the physical therapy setting. Phys Ther 2008;88:1322-35.

48. Rafiq S, Zia S, ljaz MJ, et al. Role of weight-bearing exercises in the treatment of post-menopausal osteoporosis. J Coll Physicians Surg Pak 2018;28:122-5. 